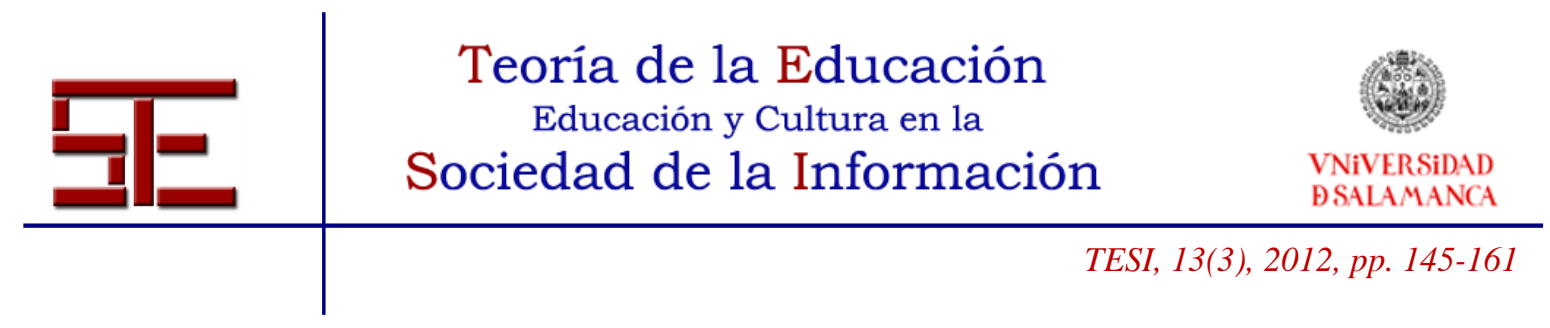

\title{
LABORATORIO MÓVIL TECNO EDUCATIVO: CURSOS DE ROBÓTICA DE BAJO COSTO PARA LA ALFABETIZACIÓN CIENTÍFICA Y TECNOLÓGICA
}

Resumen: La robótica educativa es un área multidisciplinaria de la ingeniería la cual ha sido un elemento invaluable para fomentar la ciencia y la tecnología como algo divertido. Sin embargo, los altos costos de su implementación como la dificultad de entrenamiento para los instructores la han hecho cada vez más común únicamente en países del primer mundo donde se cuenta con más presupuesto para su difusión. Es por eso que nace el concepto del Laboratorio Móvil Tecno Educativo, el cual tiene como fundamento la aplicación de cursos de robótica basados en la filosofía BEAM, a su vez incorpora prácticas de ciencias y prácticas cognitivas. El objetivo del proyecto es el de crear un nuevo concepto de aprendizaje robótico democrático a través del diseño y desarrollo de una plataforma tecno-educativa para niños y jóvenes con la finalidad de fomentar la alfabetización científica y tecnológica para el desarrollo de conocimiento y pensamiento analítico y científico. La evaluación de los cursos muestran una mejora del $36 \%$ respecto al conocimiento de ciencias y matemáticas, lo que demuestra la necesidad de incorporar un curso de robótica dado a un nivel general público.

Palabras clave: Cursos de robótica; robótica educativa; LMTE (Laboratorio Móvil Tecno Educativo); beneficios de la robótica en el aula.

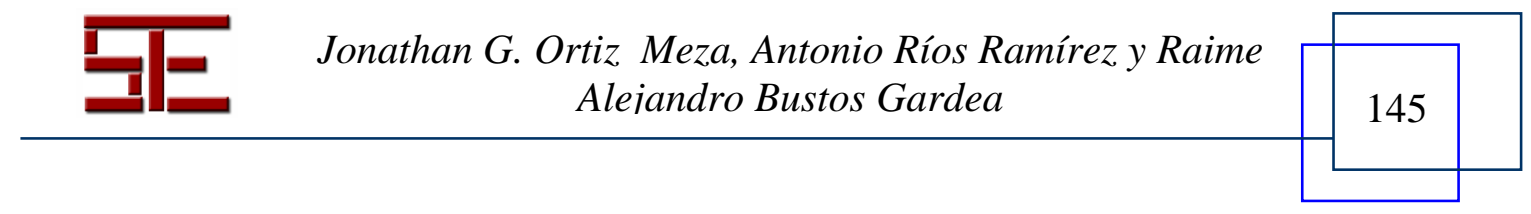




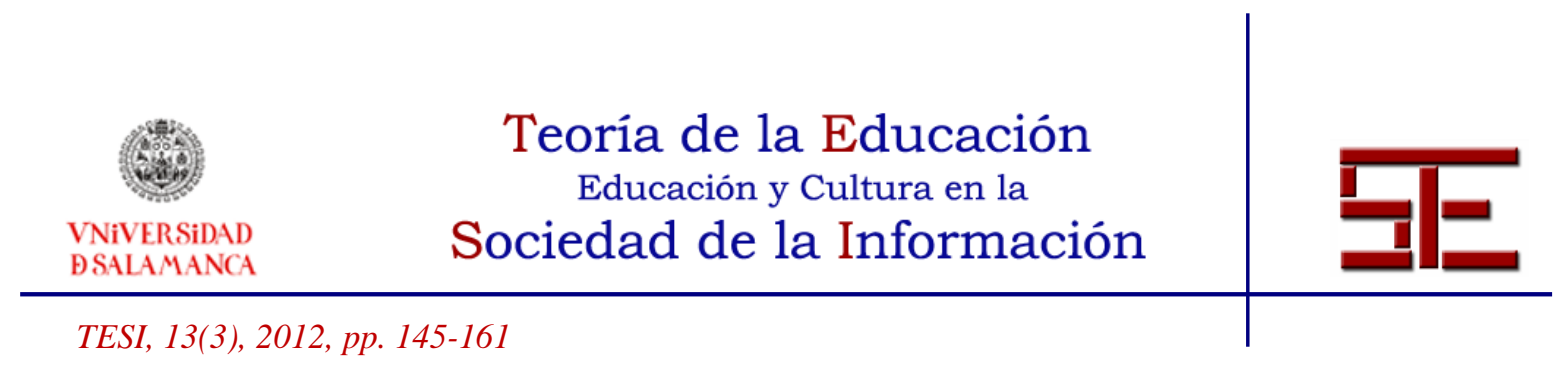

\title{
EDUCATIONAL TECHNO MOBILE LABORATORY: ROBOTICS COURSES FOR LOW COST SCIENTIFIC AND TECHNOLOGICAL LITERACY
}

\begin{abstract}
The robotics in education is a multidisciplinary branch of the engineering that has been an invaluable asset to promote the science and the technology as something fun. However, the high costs for the implementation as also the difficulty to train instructors have made the robotics more common only in first world countries, because generally they have more educational budget for this practices. For this reason the Educational Techno Mobile Lab was created; this Lab incorporates robotics courses based on BEAM robots and also sciences and cognitive practices. The objective of the Project is to create a new concept of learning based on a techno educative platform with the finality to promote the scientific and technological literacy to the development of knowledge and critical scientific thinking. The results of the evaluation present a $36 \%$ of improvement related science and technology knowledge on fourth grade students; these results show the necessity to incorporate a given robotic course to all the public education level.
\end{abstract}

Keywords: Robotics courses; Educational Techno Mobile Laboratory; benefits of the robotics.

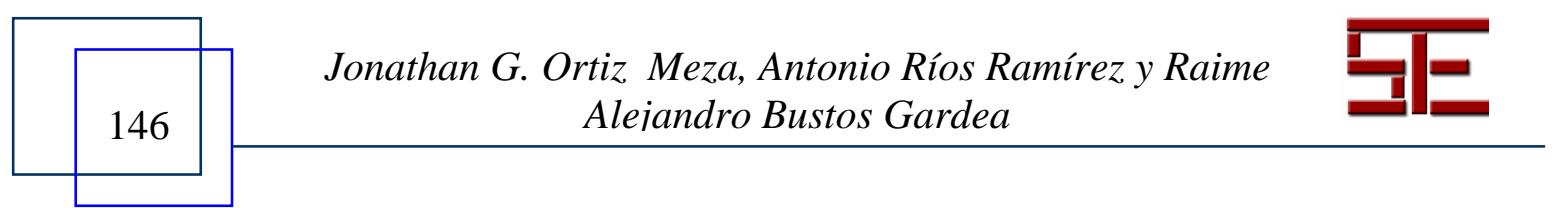




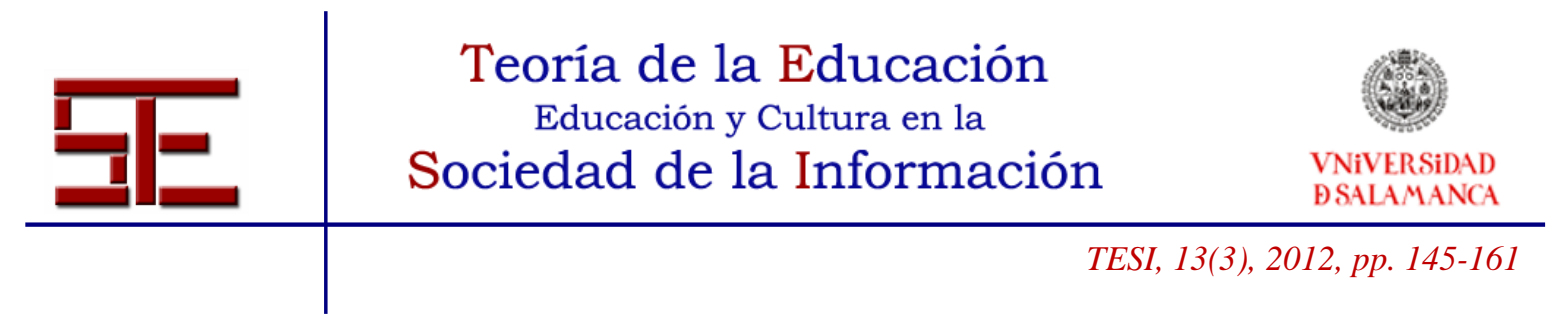

\section{LABORATORIO MÓVIL TECNO EDUCATIVO: CURSOS DE ROBÓTICA DE BAJO COSTO PARA LA ALFABETIZACIÓN CIENTÍFICA Y TECNOLÓGICA}

Fecha de recepción: 04/06/2012; fecha de aceptación: 05/09/2012; fecha de publicación: 30/11/2012

Jonathan Germain Ortiz Meza

jonathang.ortiz@itesm.mx

Instituto Tecnológico y de Estudios Superiores de Monterrey, Campus Chihuahua

Antonio Ríos Ramírez

antonio.rios@itesm.mx

Instituto Tecnológico y de Estudios Superiores de Monterrey, Campus Chihuahua

Raime Alejandro Bustos Gardea

raime.bustos@itesm.mx

Instituto Tecnológico y de Estudios Superiores de Monterrey, Campus Chihuahua

\section{1.- INTRODUCCIÓN}

A principios de los 80's, la robótica educativa empieza a ser usada de distintas formas para motivos educacionales, en especial porque promueve la ingeniería y la ciencia como algo divertido (Malec, 2001). A causa de los enormes beneficios demostrados, tales como trabajo en equipo, fomento de la creatividad, motivación, autoidentificación con la ciencia y la ingeniería, habilidades para la resolución de problemas, entre otros (Ortiz, 2011), existe una tendencia mundial a incluir la robótica como una herramienta para la educación. Países como Japón, China, Estados Unidos, Australia, Suecia, entre otros, utilizan esta actividad con gran frecuencia para reforzar su sistema educativo. El mercado comercial de los robots educativos está creciendo enormemente a nivel mundial. Un estudio reciente por la Japan Robotics Association, United Nations Economic Commision and International Federation of Robotics indica que el crecimiento del mercado de robots personales, incluyendo aquellos usados para entretenimiento y propósitos educativos, ha sido colosal. Y que esta tendencia podría continuar en las siguientes décadas. (Williams; Ma; Prejean; Ford \& Lai, 2008).

Para poder comprender por qué se hace un énfasis en la robótica en el aula y poder definir el concepto en sí, es necesario hondar un poco en algunos aspectos de la educación. Países, instituciones e individuos están enfrentándose a retos significativos

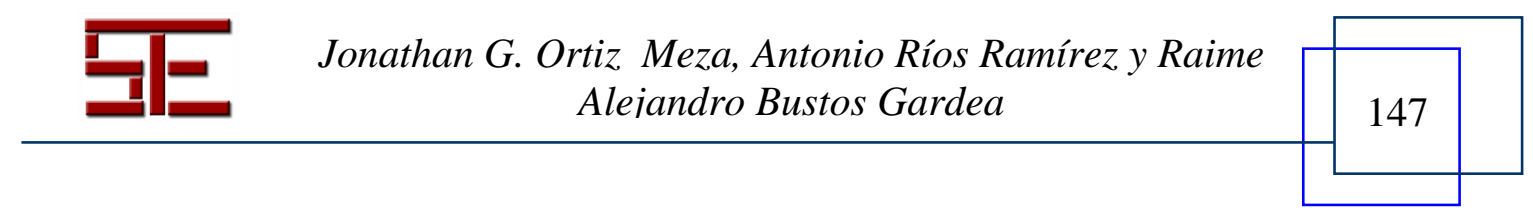




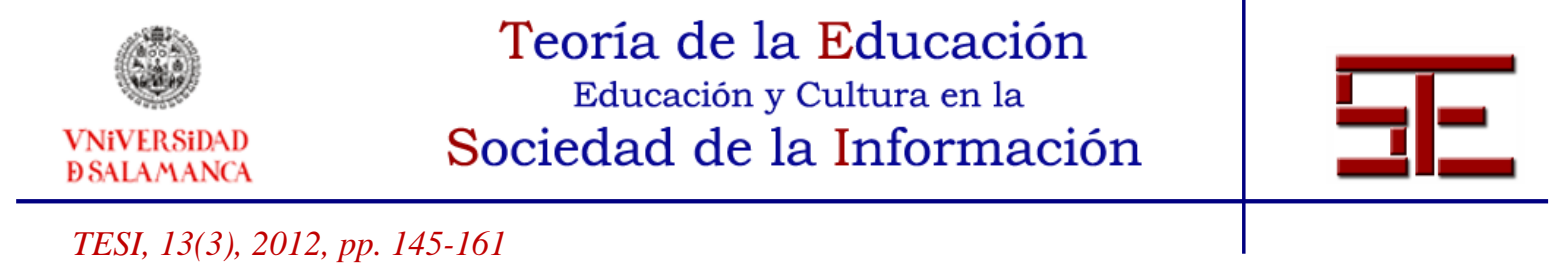

lo que respecta a los sistemas de educación (Buiu, 2008). Existe una necesidad inherente de un sistema de aprendizaje flexible, ante esta necesidad de buscar nuevas técnicas de aprendizaje, existe hoy en día un énfasis en mejorar los modelos educativos con base en modelos prácticos, los cuales fomenten la adquisición del conocimiento de una forma tangible para el alumno. En donde el conocimiento que se imparta esté basado en modelos teórico - prácticos, es decir, buscar un equilibrio en la teoría impartida así como también en la práctica de la misma donde se fortalezca el conocimiento en el alumno.

Aunado a esto, se presenta el factor de que las nuevas generaciones son influenciadas por la tecnología y la era digital. Se define a la generación como nativa de la tecnología a aquella que por el hecho de haber nacido luego del auge digital de los años 1980 y 1990 hace que se adapten de manera extraordinaria a futuras tecnologías como ninguna otra generación. Se dice que esta generación es el $18 \%$ de la población mundial, por lo que su influencia en el contexto social y educativo resulta muy importante de considerar. (Tapscott, 2008).

El sistema educativo bajo el esquema de educación tomado como un todo, es un depósito de varios procesos y procedimientos. En esta educación se presenta una depreciación sistemática del interés del alumno por el estudiar, agregándose a esta depreciación la necesidad de fomentar la práctica. Esto se engloba en una separación entre el conocer y el hacer, la teoría y la práctica, entre la mente como el fin y el espíritu de la acción como el cuerpo. Es así como Dewey comparte que la teoría del conocimiento debe derivar de la práctica, lo cual es lo más exitoso en la producción de conocimiento; y es de esta forma que la teoría será reforzada para mejorarse en los métodos los cuales la hacen menos exitosas. (Dewey, 1916).

Un modelo teórico - práctico, que cumple con las necesidades de integrar el conocer y el hacer como lo menciona Dewey y abarca los requisitos tecnológicos de las nuevas generaciones es la incorporación de la robótica en el aula de clases, la cual es un campo de la ingeniería interdisciplinaria e inherente que conforma las ingenierías eléctrica, mecánica y ciencias de la computación, así como matemáticas, físicas, sistemas de ingeniería y en algunas instancias psicología, neurociencia cognitiva y filosofía (Robinette et al., 2009). La amplitud de los problemas presentados por la robótica motiva el desarrollo de la integración de conocimiento y métodos de solución de problemas desde diferentes rangos de aproximación. El estudio de la disciplina de la

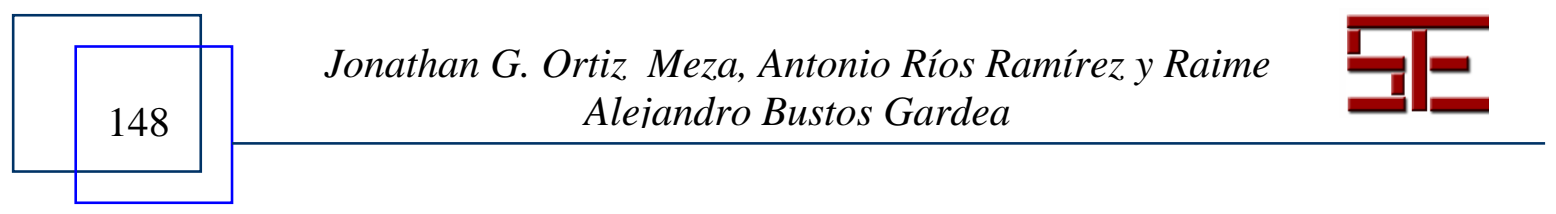




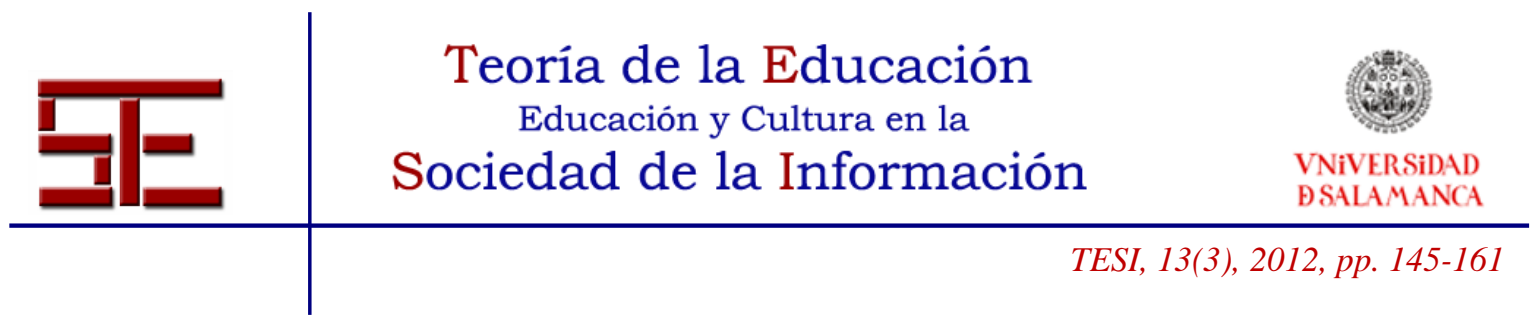

robótica puede dar a los alumnos una valiosa perspectiva en la integración de sistemas, así como experiencia en área del mundo real de la resolución de problemas.

Sin embargo, se afirma que las transferencias de tecnología y la organización o enfoque de los currículos son a menudo copia de esquemas extranjeros que ignoran la idiosincrasia, la cultura y las necesidades de la comunidad concreta. Los currículos académicos ignoran el estudio de la realidad socioeconómica del país y la discusión de sus problemas, imposibilita la formación de ciudadanos creados, dinámicos y conscientes de su ambiente social (Suárez, 2002).

El presente artículo tiene como intención el dar a conocer el trabajo del Laboratorio Móvil Tecno Educativo, el cual está basado en cursos de robótica y robots BEAM, como también en prácticas de ciencia y prácticas cognitivas, el Laboratorio se implementa en Chihuahua, México, con la intención de ofrecer cursos de robótica gratuitos a más de 1000 estudiantes de los grados educativos primaria, secundaria y preparatoria.

El presente artículo está organizado de la siguiente forma: la parte II hace hincapié en conceptos de la robótica y la educación contemporánea. La sección III describe el concepto y la composición de los cursos del Laboratorio Móvil Tecno Educativo. La sección IV muestra los resultados de la evaluación en la primera iteración del curso. Finalmente, la sección $\mathrm{V}$ señala las conclusiones del trabajo realizado.

\section{2.- LA ROBÓTICA Y LA EDUCACIÓN CONTEMPORÁNEA}

Se dice que se vive en una era tecnológica. En este nuevo ambiente social, las comunicaciones y las tecnologías de la información se han convertido en una necesidad que ha marcado el desarrollo de la sociedad, desde cambios culturales hasta comportamientos sociales a través del tiempo (Thomaz; Aglae; Fernandes; Pitta; Azevedo; Burlamaqui, et al., 2009). Es obvio que la tecnología también envuelve a la educación, la cual se está involucrando cada vez más en instituciones educativas. Es de esta forma que la comunidad académica está sufriendo un proceso organizacional teniendo como consecuencia construir mejores ideas relacionadas con la tecnología y la educación.

Según Saracho (2008), el conocimiento acerca de ciencia y tecnología es un requerimiento importante para toda sociedad contemporánea actual. Un número

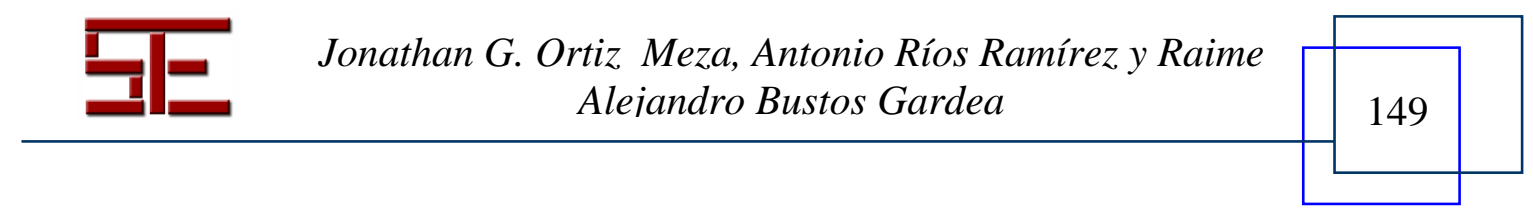




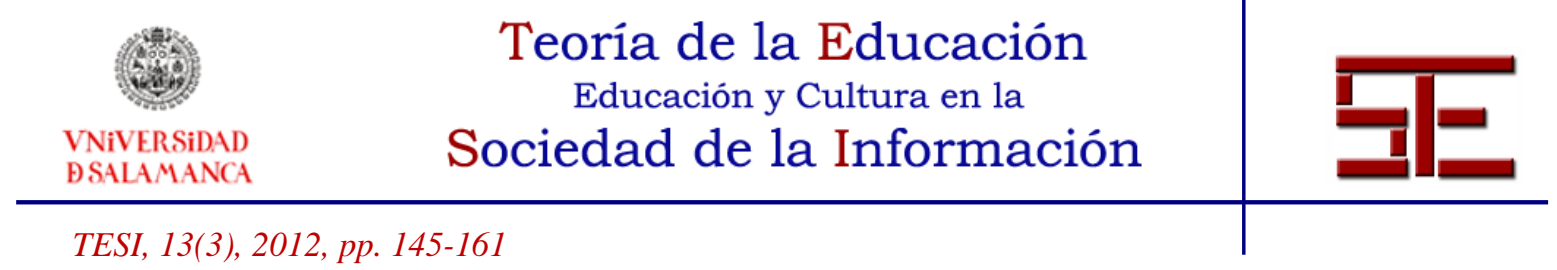

incremental de profesiones requieren el uso de conceptos científicos y habilidades tecnológicas, los cuales dependen del conocimiento científico. A su vez se dice que el conocimiento científico y tecnológico debe formar parte de cada individuo en la sociedad. El pensamiento científico y las habilidades de resolución de problemas así como las habilidades tecnológicas son necesarios para el desarrollo de la sociedad.

El robot es una nueva forma de aparato tecnológico que cada vez está más presente en los ambientes educacionales. Un robot educativo se puede definir como un aparato electro-mecánico o computacional que es capaz de interactuar con el medio ambiente y con personas ejecutando acciones predefinidas o funciones autónomas. A su vez se menciona que es una forma muy buena para desarrollar y de estimular el trabajo como instrumento motivacional si es usado dentro del proceso de enseñanza y aprendizaje (Thomaz et al., 2009). Los robots han encontrado su camino en medio del salón de clases, es más sobre educación que enseñar robótica, ya que a través de estos se ayuda a enseñar ciencia, matemáticas, mecánica, trabajo en equipo e incluso habilidades de administración. Incluso se menciona que está muy orientada a resolver problemas y a su vez convencer a los estudiantes de cualquier grado de que es interesante y útil (Vitko, Jurišica, Babinec, Duchoñ \& Klúcik, 2010).

Desde los finales de los 80's, con el comienzo del curso de robótica del Massachusetts Institute of Technology (MIT), titulado -6.270-, se dice por parte de Malec (2001) que los robots han sido usados en la educación de muchas formas y propósitos. Sin embargo, Ruiz-Velasco, Beauchemin, Freyre, Martínez, Valentina, Rosas, et al. comentan que es a partir de 1975 que aparece el primer intento de experiencias de uso de robots en el aula (2006). Por otra parte, se puede agregar a manera de antecedentes que el uso de la robótica como apoyo a la educación nace con los esfuerzos de Seymour Papert en la década de los 60's, quien trabaja la relación ciencia, adquisición de conocimiento y la mente infantil con el uso del recurso de la computadora en el proceso de aprendizaje, siendo estos esfuerzos los inicios de la informática educativa. Es de aquí que nace el lenguaje de programación Logo, que subsiguientemente se convertiría en la base de los robots pedagógicos Lego Mindstorms (Papert, 1993). Con el paso del tiempo se crearon una gran variedad de productos e instrumentos de robótica para el aula de clases o con fines lúdicos, tal es el ejemplo de los robots bajo la filosofía B. E. A. M. (acrónimo del inglés Biology, Electronics, Aesthetics and Mechanics) por Mark Tilden, que, a diferencia de los Lego Mindstorms, no se necesita una computadora para su creación y funcionamiento (Tilden \& Hrynkiw, 2002).

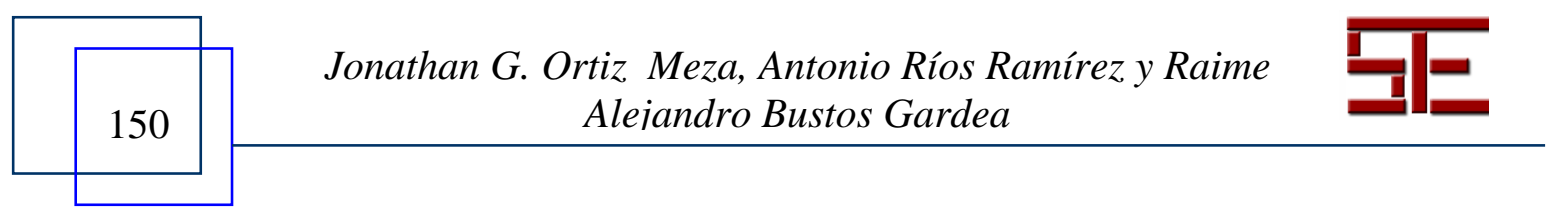




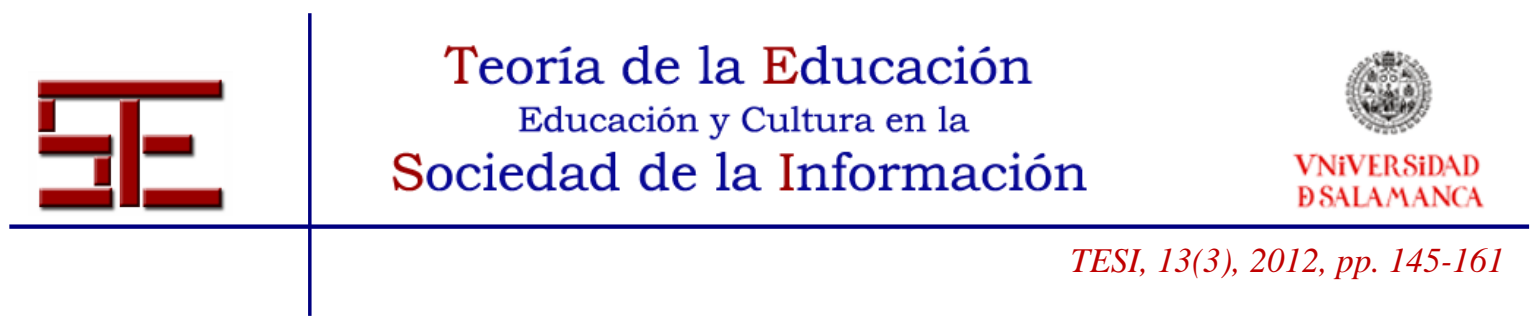

Bajo este contexto se pueden dar a conocer dos conceptos diferentes, pero a la vez tan similares: la robótica educativa y la robótica pedagógica. Malec lo diferencia de una forma más singular: "La robótica en la educación" y "La robótica para la educación". Donde en la robótica en la educación, los robots juegan un papel aparte de ser ingenieriles y con contenido de ciencia, también involucra la diversión. Mientras que en la robótica para la educación los robots son usados para procesos educacionales.

Sánchez (2003) menciona que algunos autores consideran la robótica pedagógica como la evolución de la informática educativa, ya que bajo este contexto se empezaron a explorar nuevos modelos educativos y detonaron el concepto de la robótica en el aula de clase. Ruiz-Velasco a través de Vivet (1990) da la siguiente definición de robótica pedagógica: "Una actividad de concepción, creación/puesta en práctica, con fines pedagógicos, de objetos técnicos físicos que son reducciones bastante fiables $\mathrm{y}$ significativas de procedimientos y herramientas robóticas realmente utilizadas en la vida cotidiana, particularmente en el medio industrial". Por otra parte, Trujillo (2007) nos comparte que la robótica educativa es un escenario que permite al infante o joven construir su propio conocimiento a partir de una posición del método científico; permitiéndoles aprender de una forma más práctica, sencilla y dinámica, donde se logra que los usuarios sean creadores e investigadores y no meramente consumidores de tecnología.

Por definición del presente autor se puede decir que la robótica pedagógica se vale del desarrollo de laboratorios virtuales donde la creación de conocimiento e interacción es a través de la computadora, y la robótica educativa tiene su área de conocimiento en la construcción o armado de robots que no necesariamente están unidos a una computadora para su funcionamiento, como los robots B. E. A. M. mencionados anteriormente. Sin embargo, se observa que ambas disciplinas conducen a una nueva forma de transmitir conocimiento e integración de distintas áreas o disciplinas a través de otras ciencias, dando así al usuario una nueva forma de educación a través de un modelo físico o virtual de interacción.

Alimisis y Kynigos (2009) comparten que en los últimos años el interés en utilizar la robótica se ha incrementado y se han realizado muchos intentos a nivel mundial de introducir la robótica en la educación desde pre-escolar hasta profesional, principalmente como una actividad interdisciplinaria de aprendizaje enfocada en las materias como ciencia, matemáticas, informática y tecnología. Sin embargo, la introducción satisfactoria de la innovación educativa en la escuela no es sólo cuestión

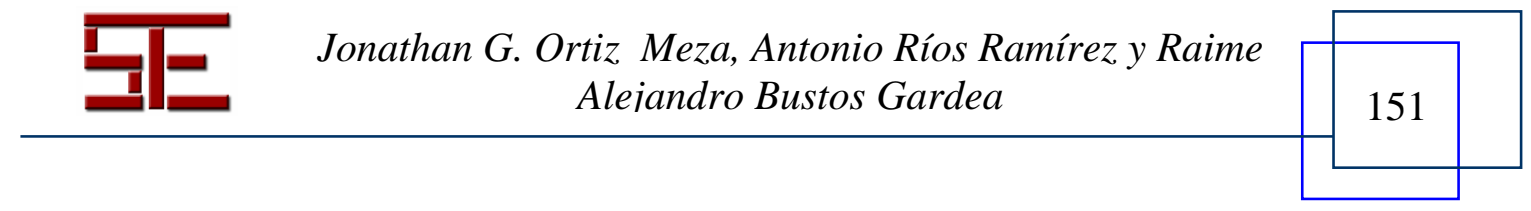




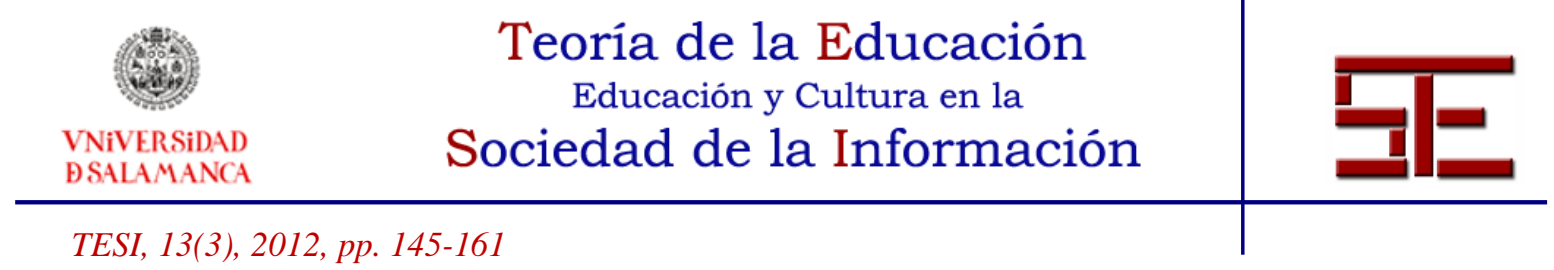

de tener nuevas tecnologías. La tecnología por sí sola no puede ayudar a los estudiantes a aprender directamente ni cambiar su forma de pensar. Los factores importantes para una adecuada implementación en cualquier ambiente educativo para el éxito de la innovación son: una filosofía educativa, un currículo y un ambiente de aprendizaje adecuados (Alimisis, 2009).

Con el rápido desarrollo de la ciencia y la tecnología se provee un mejor ambiente educacional tanto para maestros como alumnos. La robótica es una disciplina integral que interactúa con muchas áreas de estudio como lo son: Ingeniería Eléctrica, Ingeniería Electrónica, Ingeniería Mecánica, Inteligencia Artificial, Sistemas computacionales, entre otras. Además, el trabajo práctico de la robótica a nivel educativo puede ayudar a los estudiantes a desarrollar las habilidades necesarias y de comunicación para trabajo en equipo (Xuemei; Jin-hu \& Lixing , 2009).

Las nuevas demandas de la sociedad han estimulado al desarrollo de un nuevo tipo de educación. Así bien, Xuemei et al., comparten que la educación debería estar orientada a entrenar personas especializadas en tecnología educativa, para llevar con ello investigación y cursos, explorar y aplicar varios tipos de tecnología. Existen investigaciones que demuestran que los estudiantes que han obtenido muy buenas calificaciones en sus grados escolares no son competentes en solucionar problemas de la vida real o problemas prácticos fuera del salón de clases (Guoqing, 2003).

Para resolver este problema, precisamente una de la soluciones es el cultivar a los estudiantes para llegar a ser buenos en aprender y adaptarse a través del aprendizaje colaborativo. La robótica en el aula ayuda no sólo a cultivar a los estudiantes en tomar y usar el conocimiento o habilidades, sino también para descubrir problemas y resolverlos de una manera activa. El adaptarse a los ambientes y sobre todo el aprender cómo aprender.

\section{3.- LABORATORIO MÓVIL TECNO EDUCATIVO}

El Laboratorio Móvil Tecno Educativo tiene como fundamento la aplicación de cursos de robótica basados en la filosofía BEAM, cuyo precio de los modelos utilizados está en el rango entre los 80 a 250 pesos mexicanos (alrededor de 6 a 20 dólares americanos), a su vez incorpora prácticas de ciencias y prácticas cognitivas. El objetivo del proyecto es el de crear un nuevo concepto de aprendizaje robótico democrático a través del diseño y desarrollo de una plataforma tecno educativa para niños y jóvenes con la finalidad de

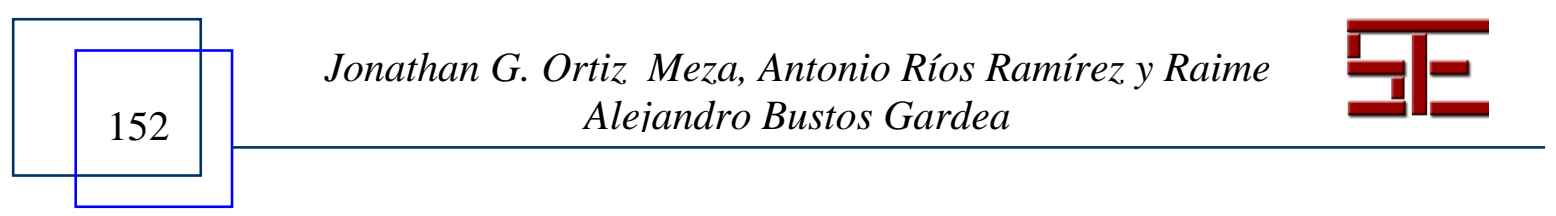




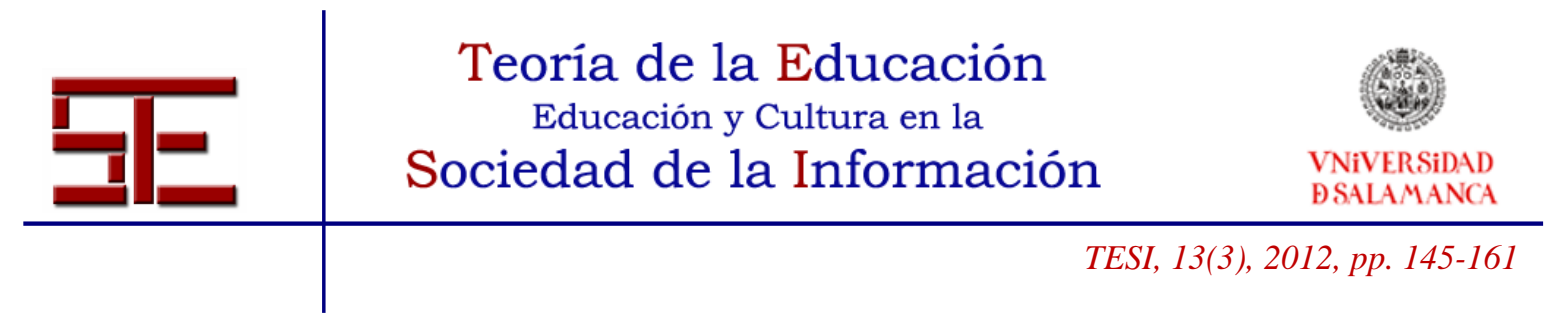

fomentar la alfabetización científica y tecnológica para el desarrollo de conocimiento y pensamiento analítico y científico.

En el grado educativo primaria, el tamaño de los grupos participantes varía entre el rango de 23 a 35 estudiantes. Para la impartición de los cursos se diseñaron dos niveles de 8 sesiones con duración de hora y media cada una. Los temas del primer nivel incluyen:

1) Introducción a la Robótica.

2) Seguridad y uso de herramientas.

3) Armado del Robotemblorín ${ }^{\text {MR }}$ (Robotech, 2012).

4) Introducción a la Electrónica y práctica de los limones.

5) Robot Apache ${ }^{M R}$.

6) Práctica Cognitiva.

Por parte del segundo nivel, los temas que se desarrollan son:

1) Introducción a la filosofía BEAM.

2) Práctica con resistencias.

3) Circuito de la destreza.

4) Robot BEAM Zoobot ${ }^{\text {MR }}$ (Robotech, 2012).

5) Práctica Cognitiva.

\section{1.- Prácticas de ciencias}

La idea de incorporar las prácticas de ciencias nace del concepto de que un curso de robótica tiene que tener una relación directa con las ciencias, es así que se incorporan dichas prácticas que están directamente relacionadas a temas de ingeniería, un ejemplo es la práctica de los limones, la cual el alumno es responsable de llevar su material para el experimento el cual es un limón, un clavo y una lamina de fusible. El objetivo del experimento es que después de la sesión de electrónica, donde se explican los distintos tipos de energía, el alumno comprenda mediante la práctica de donde obtiene el ser humano la energía, eso intrínsecamente ligado a la comprobación de que las frutas y verduras proveen energía, pero ahora el alumno lo comprueba con la ayuda de un multímetro.

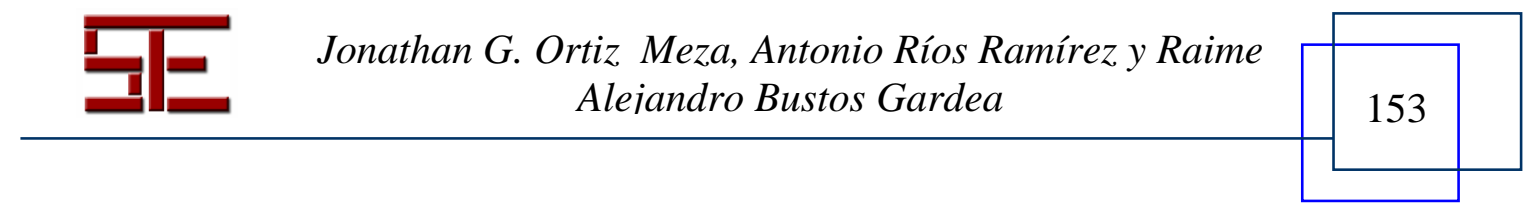




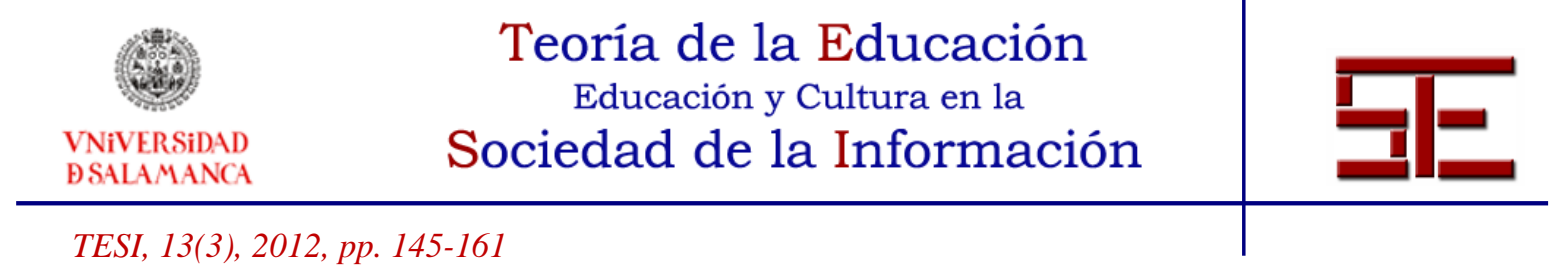

\section{2.- Prácticas cognitivas}

Las prácticas cognitivas nacen del concepto de Dewey, el cual comparte que hay una enorme necesidad de llevar la teoría a la práctica, así bien, dado que el robot es un medio motivador el cual puede ser utilizado para fomentar de una forma divertida las matemáticas, las prácticas cognitivas están conceptualizadas y diseñadas para llevar a cabo operaciones sencillas de matemáticas mediante el uso del robot. Por ejemplo, una vez que el niño termina el robot, por equipos se hace una competencia donde tienen que tomar los tiempos que el robot recorre cierta distancia y con ello tomar los promedios de las vueltas. Mediante esta interacción con el robot y las matemáticas el alumno comprende la utilidad práctica de sus asignaturas de su clase. Otro ejemplo se da en el armado del Robotemblorin, el cual tiene de cuerpo un vaso de plástico de $12 \mathrm{~cm}$ de diámetro, al alumno se le explica lo que es el punto de equilibrio y con la ayuda de un transpordador y un compás el alumno debe partir el diámetro en tres partes iguales donde futuramente pegará las patas del robot.

\section{3.- Robots utilizados}

Para el desarrollo de los cursos se utilizaron robots basados en la filosofía BEAM. Como se explica con anterioridad, estos robots son de bajo costo y tienen su fundamento en asimilar el mundo de los insectos al mundo de los robots a través de elementos simples de la electrónica analógica.

Este concepto fue creado por Mark Tilden y ha sido muy popular en el medio ya que los robots pueden crearse de deshechos electrónicos y materiales simples. Los robots BEAM se clasifican como reactivos, es decir, no necesitan ser programados para simular un comportamiento sino que su lógica de funcionamiento se encuentra embebida en su diseño e implementación. Por ejemplo, se vale del uso de resistencias, capacitores, motores, sensores y algunos componentes digitales como lo son los flip flops o temporizadores. Pero lo que sí es un hecho es que estos robots no utilizan componentes de memoria como lo es un microprocesador o una ROM, en adición estos robots consumen menos energía y se usan en ocasiones celdas solares para su funcionamiento generando robots autosustentables para su funcionamiento.

Es así que, a través de estos robots, los estudiantes exploran conceptos básicos de electrónica como también de los componentes usados y además conceptos básicos de

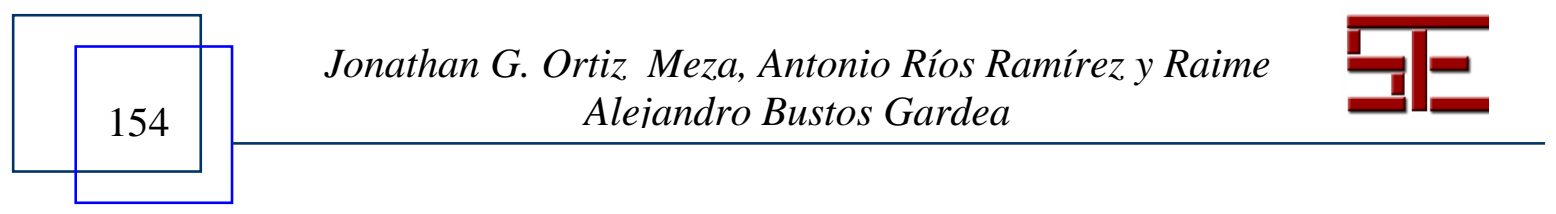




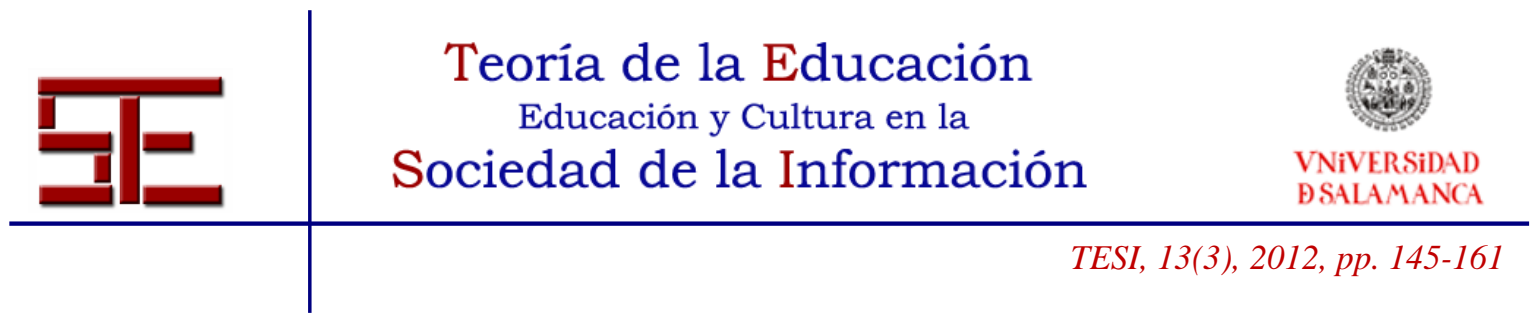

mecánica para el funcionamiento del robot como lo son engranes, punto de equilibrios, entre otros.

En la ejecución de los cursos, los estudiantes trabajan en parejas, compartiendo herramientas y ayudándose en tareas comunes para llevar a cabo las actividades de la sesión. Los estudiantes aprenden a:

1) Reconocer y saber para qué se usan los componentes electrónicos y mecánicos que se utilizan en los distintos robots de los cursos (resistencias, motores, switches, etc.), así como también otros tópicos como el saber leer el valor de una resistencia, polaridad de los componentes, entre otros.

2) Se aprende a cómo manejar herramientas como el cautín, multímetro y distintos tipos de pinzas, también la importancia de trabajar con cuidado al aprender los principios de seguridad.

3) Poner en práctica conceptos simples por medio de prácticas cognitivas de matemáticas tales como sumas, promedios y otros más complejos como el uso de diámetros y ángulos.

4) Aprendizaje de temas relacionados a tipos de energía y conceptos básicos de electrónica y robótica.

Con el objetivo de capacitar a los instructores y tener una base estandarizada de información para el estudio de los cursos, se decide utilizar un repositorio electrónico de conocimiento basado en la filosofía wiki, el cual permite no solamente el estudiar y obtener el material necesario para impartir el curso, sino también su mejora continua a través de la retroalimentación de los profesores. El sitio puede ser visitado en www.wikibotics.org .

Los instructores de los cursos son alumnos de las carreras de electrónica y mecatrónica del Instituto Tecnológico y de Estudios Superiores de Monterrey, Campus Chihuahua. Dichos alumnos cursan entre el quinto al noveno semestre de la carrera. Para la impartición de los cursos, se decide emplear a un instructor por cada quince estudiantes para garantizar una atención de calidad y seguimiento adecuado a las actividades realizadas.

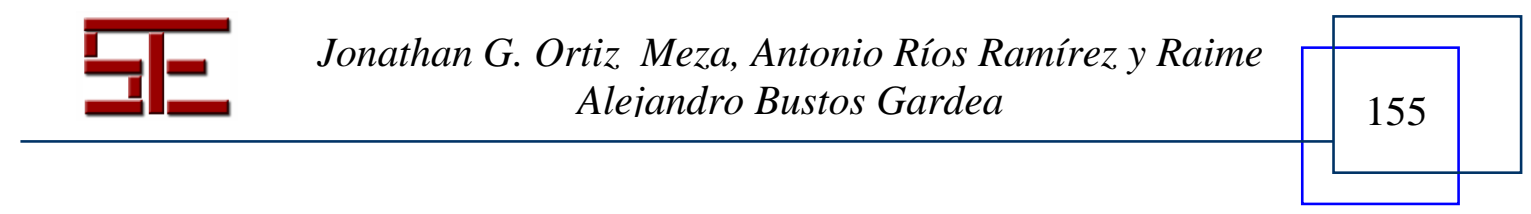




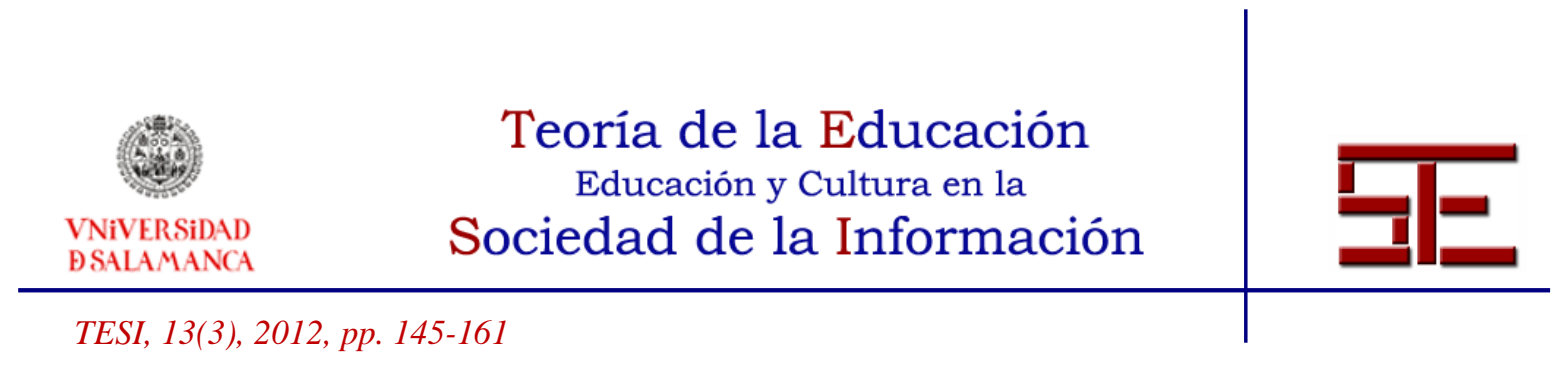

\section{4.- IMPLEMENTACIÓN Y EVALUACIÓN DE LOS CURSOS}

Por motivos de la implementación de los cursos del Laboratorio Móvil Tecno Educativo se define el cuarto año de primaria como el objeto de estudio. En total fueron 16 escuelas primarias las participantes, pero por motivos de alcance en esta sección se da a conocer los resultados de 6 de ellas. La evaluación parte desde el concepto de tener un grupo de aplicación, el cual se define como aquel grupo que cursa robótica y el grupo de control, el cual es aquel grupo que no cursa robótica. El sustento estadístico para dar validez a la presente evaluación se explica que a partir de un universo de 18,000 estudiantes de cuarto grado de primaria en la ciudad de Chihuahua y un intervalo de confianza del 5\%, se tiene una muestra mínima de 377 alumnos como mínimo.

Ortiz et al. (2011) señalan que se aplican dos tipos de exámenes para la evaluación de los cursos, el primero de ellos, el examen de ciencias e ingeniería, consta de un examen de 44 preguntas abstraídas del examen Internacional TIMMS (del acrónimo del ingles: "Trends in International Mathematics and Science Study", Estudio Internacional de Tendencias en Matemáticas y Ciencias) y del examen mexicano ENLACE (Evaluación Nacional del Logro Académico en Centros Escolares) y mediante una serie de fórmulas se genera el Índice de Ciencias e Ingeniería (ICI), cabe mencionar que para el examen ICI las preguntas no están relacionadas al material que se imparte en el curso, es decir, que se presenta una evaluación indirecta con base a preguntas de ciencias y matemáticas. Respecto a la otra evaluación está relacionada con medir la percepción de los beneficios de la robótica mediante una encuesta de 22 preguntas con base a una escala Likert del 1 al 5. A través de este instrumento se presenta el Índice de Percepción de Beneficios (IPB), el cual es generado a través del cómputo de las variables expuestas por Ortiz (2011) en relación al Modelo de la Robótica en el Aula.

Así bien, las variables evaluadas en el ICI son: Física, Matemáticas, Electrónica, Mecánica, Informática, Ciencias de la Computación y programación por parte del IPB se evalúan los beneficios tales como: Creatividad, Trabajo en Equipo, Resolución de Problemas, Motivación y Autoidentificación con la Ciencia y la Tecnología.

Es importante recordar que se realiza una comparación directa de los grupos que tomaron robótica respecto a los que no únicamente de la misma escuela. Esto es importante porque entre esos grupos existen las condiciones socioeconómicas, culturales y educativas necesarias para ver el progreso de los niños que sí toman robótica. En la Fig. 1 se muestran los resultados de las evaluaciones realizadas a 6

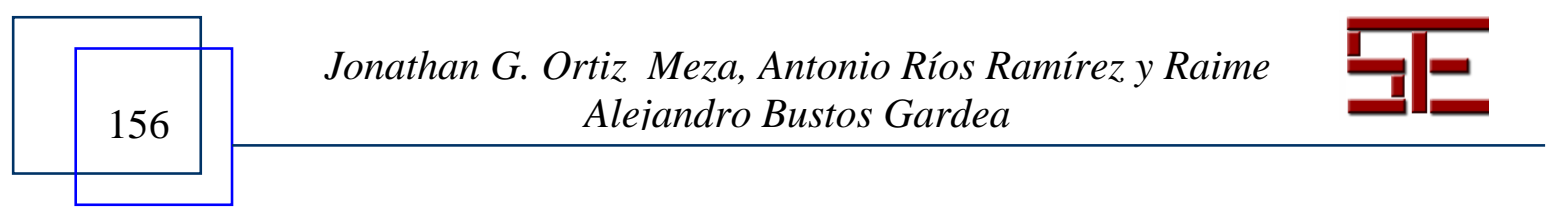




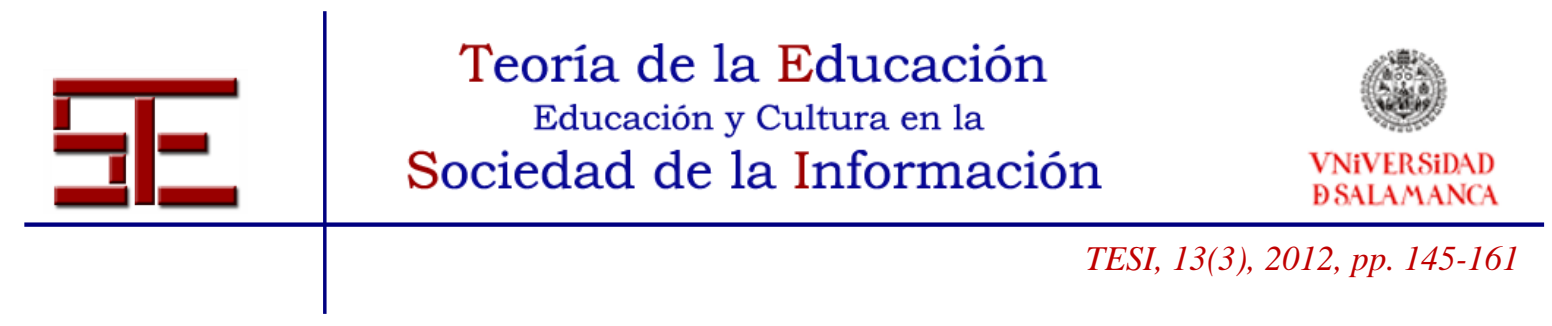

escuelas. Cabe mencionar que se presenta la mejora porcentual por escuela en ambos índices después de realizar la división del promedio de los resultados del grupo de aplicación sobre el grupo de control. De esta forma se puede observar si hay o no incremento de las calificaciones del examen o bien de la percepción de los beneficios.

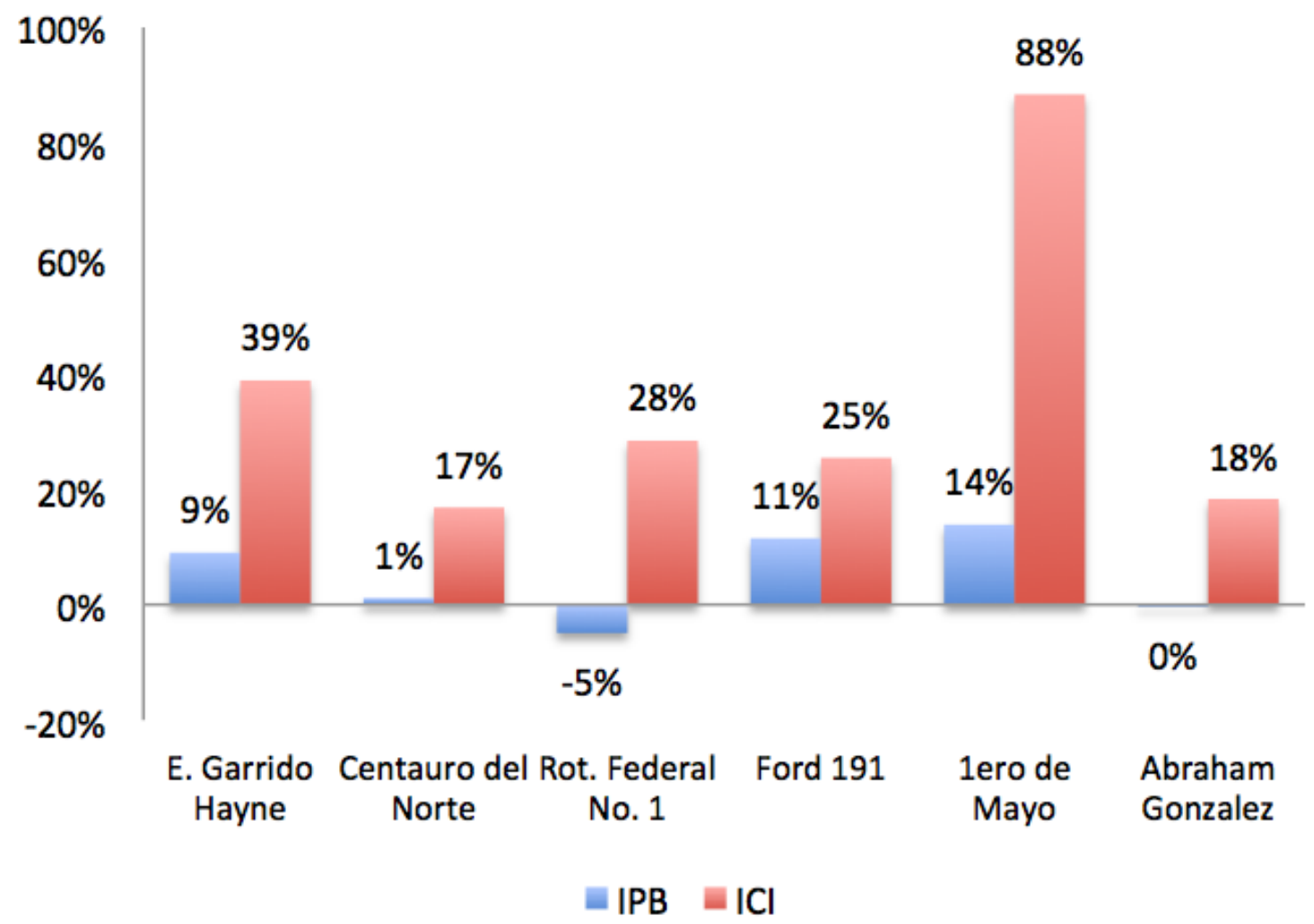

Fig. 1 Comparativo porcentual de la evaluación realizada, fuente Ortiz et al. (2011).

Como se puede observar, todas las escuelas presentan una mejora respecto a su grupo de control conforme el examen de ciencias e ingeniería. Sin embargo, esto no se presenta en el Índice de Percepción de los Beneficios, donde los datos varían. Se llega a la conclusión de que el instrumento utilizado para este índice necesita ser mejorado respecto a la sensibilidad de las preguntas.

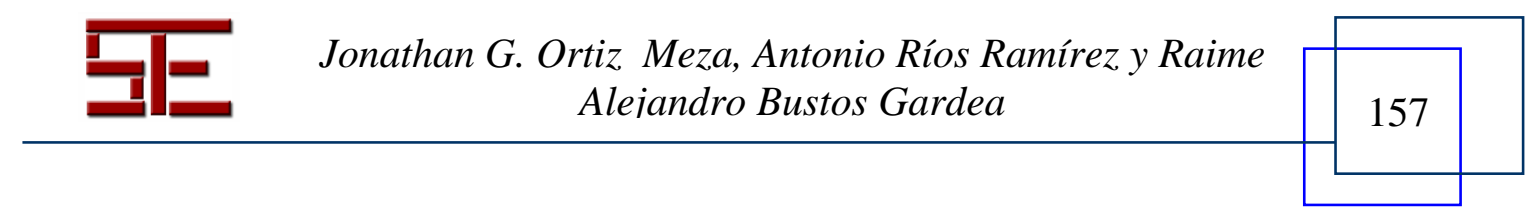




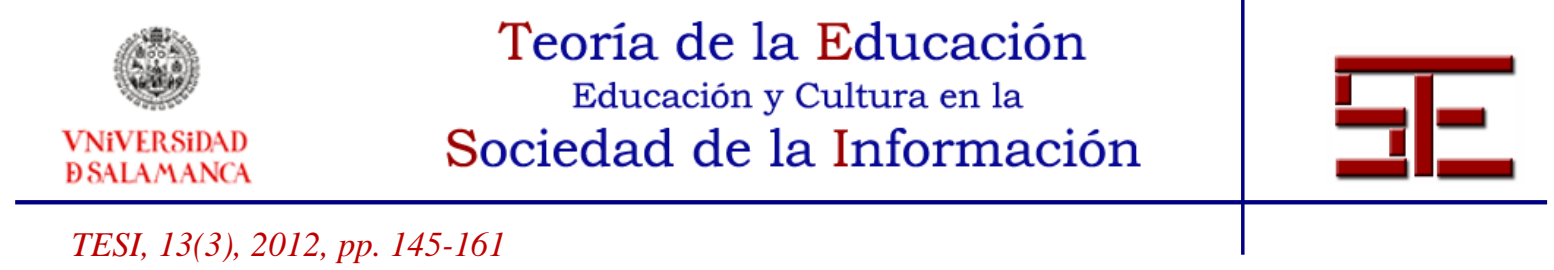

Respecto al Índice de Ciencias e Ingeniería se muestra una mejora en promedio del $36 \%$ respecto a los grupos que no tomaron robótica. El mayor contribuyente se presenta en la escuela 1ero de Mayo, donde sube hasta el $88 \%$, al analizar directamente la situación, se presentó la situación en que ambos grupos habían tenido dos exámenes de sus asignaturas regulares y al momento de aplicar el examen de ciencias e ingeniería los que sí tomaron robótica se vieron motivados a contestarlo a conciencia, mientras los que no, su interés de sacar una nota era prácticamente nulo.

\section{5.- CONCLUSIONES}

En el actual artículo se dio a conocer el trabajo aplicado del Laboratorio Móvil Tecno Educativo, en el cual, a través de cursos económicos de robótica, se lograron resultados que muestran la validez y justificación de su incorporación en las escuelas públicas. Estos resultados cuentan con sustento científico para asegurar que los estudiantes de los cursos del LMTE mejoran hasta un 36\% su comprensión de ciencias y matemáticas respecto a los grupos de control. Por lo que se asegura que a través de los cursos de robótica se puede acortar el rezago educativo de los exámenes internacionales como los son PISA o TIMMS. A su vez esta mejora en el nivel de ciencias y matemáticas representa una premisa importante para lograr la motivación de los estudiantes para que desde edad temprana logren relacionarse con temas de ingeniería y ciencias con el objetivo de que en un futuro se logre incrementar la matrícula a nivel licenciatura en estas áreas.

Por otro lado, por medio de comentarios de los docentes de grupos y observaciones directas de los instructores de robótica, se menciona que los cursos de robótica fueron un factor altamente motivante para que el estudiante logrará la empatía en incrementar su dedicación al estudio.

Dado el avance de la tecnología, es natural que los individuos y sociedades vean como una necesidad natural e intrínseca la incorporación de la misma en sus actividades cotidianas, por lo que el sistema educativo también debe sufrir un cambio evolutivo respecto a estas necesidades. La Robótica aplicada al Aula de Clases es una herramienta que permite esta evolución y cumple las necesidades educativas que nuestra sociedad demanda.

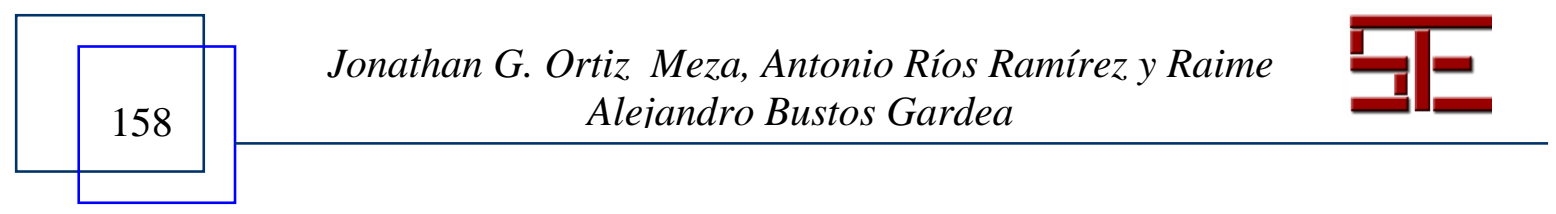




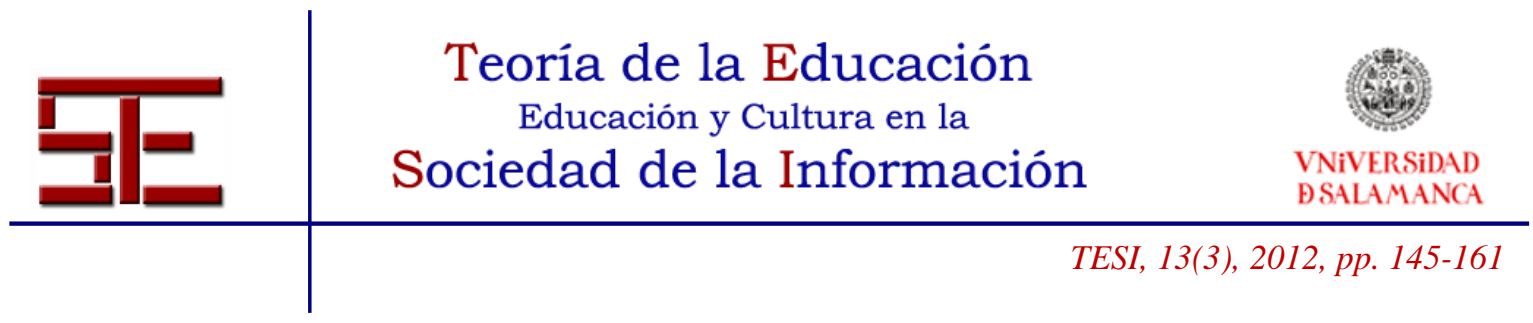

\section{6.- RECONOCIMIENTOS}

El presente autor quiere agradecer al Dr. Raime Bustos y Dr. Antonio Ríos por la confianza y su gran apoyo incondicional en la realización del proyecto del Laboratorio Móvil Tecno Educativo, como también a todo el equipo de trabajo del mismo que sin su esfuerzo y dedicación hubiera sido imposible la ejecución del proyecto.

Un agradecimiento especial a José Luis Bruni, Sergio Tejeda, Mauricio Sánchez, Olmo Moreno, Javier Rubio, Kathia Pittí y Rolando Cruz por haber fungido como expertos de la robótica educativa evaluando el Modelo de la Robótica en el Aula con su invaluable experiencia y dedicación en esta área.

Por último, se agradece ampliamente al Consejo Nacional de Ciencia y Tecnología (CONACYT), al Gobierno del Estado de Chihuahua y a la Secretaría de Educación Pública por su apoyo para la realización proyecto a través de sus representantes: Lic. Carlos Calleros y al Dr. Héctor García (CONACYT), del sistema federal de primarias a: la Ing. Marisa Tirado por su invaluable apoyo y ayuda, al Prof. Martin Ortiz la Riva y Mtra. Graciela Rodríguez y por parte del sistema estatal a: Mtra. Guadalupe Zapata y Mtra. Myriam García.

\section{7.- BIBLIOGRAFÍA}

Alimisis, A. D. \& Kynigos, C. (2009). Constructionism and robotics in education. Teacher Education on Robotics-Enhanced Constructivist Pedagogical Methods. School of Pedagogical and Technological Education (ASPETE).

Buiu, C. (2008). Hybrid Educational Strategy for a Laboratory Course on Cognitive Robotics. IEEE Transactions on Education, 51 (1), 100-107.

doi: 10.1109/TE.2007.906605.

Malec, J. (2001). Some thoughts on robotics for education. Presented at the 2001 AAAI Spring Symposium on Robotics and Education. Stanford University, March 2001.

Ortiz, J. G. (2011). Sistema de Indicadores y Metodología de Evaluación para la Robótica en el Aula (tesis de maestría). Instituto Tecnológico y de Estudios Superiores de Monterrey, Campus Chihuahua, México.

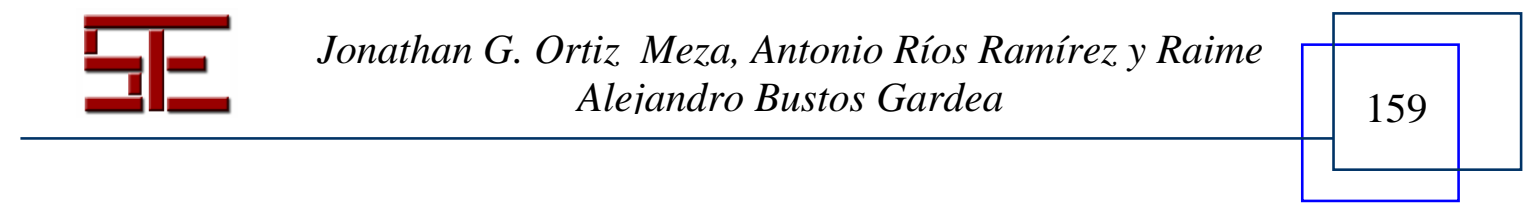




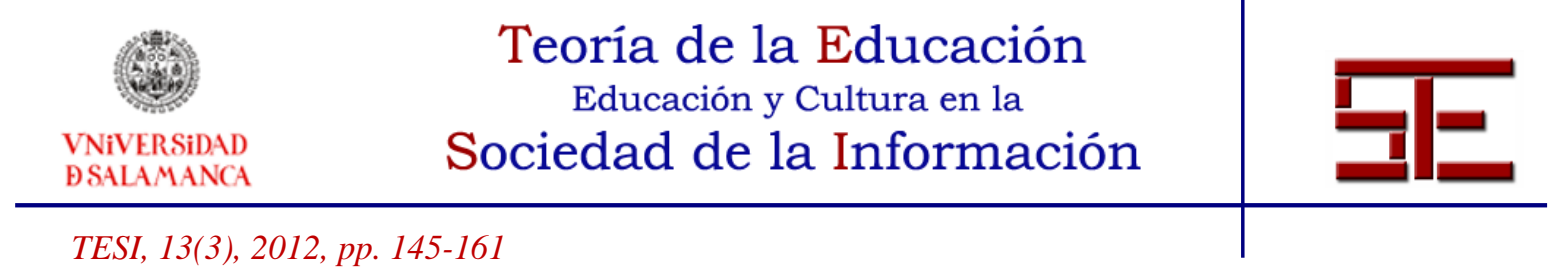

Ortiz, J., Bustos, R and Ríos, A. (2011). "System of Indicators and Methodology of Evaluation for the Robotics in Classroom". En Proceedings of 2nd International Conference on Robotics in Education (RiE 2011). (63-70). Vienna, Austria, September, 2011. . INNOC - Austrian Society for Innovative Computer Sciences.

Papert, S. (1993). Mindstorms: Children, Computers, and Powerfull Ideas. New York: Basic Books.

Robinette, P., Meuth, R., Dolan, R. \& Wunsch, D. (2009). LabRat TM: Miniature Robot for Students, Researchers and Hobbyists. En The 2009 IEEE/RSJ International Conference on Intelligent Robots and Systems (pp. 1007-1012).

Robótica Tecno Escolar de Chihuahua, Robotech. (2012). Sitio Oficial, disponible en www.robotech.com.mx.

Ruiz-Velasco, E. (2007). Educatrónica, Innovación en el aprendizaje de las ciencias y la tecnología. Distrito Federal: Díaz de Santos.

Sánchez, M. (2003). Ambientes de aprendizaje con Robótica Pedagógica (tesis inédita de maestría). Universidad de los Andes. Colombia: Bogotá.

Saracho, O. \& Spodek, B. (2008). Contemporany Perspectives on Science and Technology in Early Childhood Education. USA: Information Age Publishing Inc.

Suárez, R. (2002). La educación: teorías educativas, estrategias de enseñanzaaprendizaje. México: editorial Trillas.

Tapscott, D. (2008). Grown Up Digital: How the Net Generation is Changing Your World. New York; Mexico City: McGraw-Hill.

Tilden, M. y Hrynkiw, D. (2002). Junkbots, Bugbots and bots on wheels, building simple robots with BEAM Technology. Berkley: Osborne.

Thomaz, S., Aglaé, A., Fernandes, C., Pitta, R., Azevedo, S., Burlamaqui, A., et al. (2009). RoboEduc: A pedagogical tool to support educational robotics. 2009 39th IEEE Frontiers in Education Conference (pp. 1-6). Ieee. doi: 10.1109/FIE.2009.5350439.

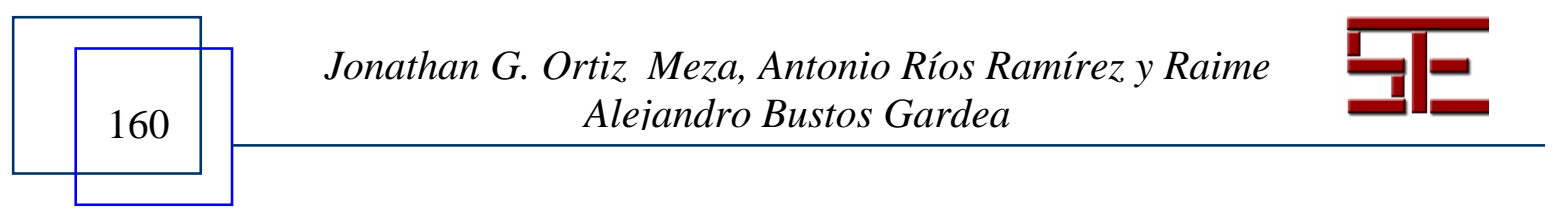




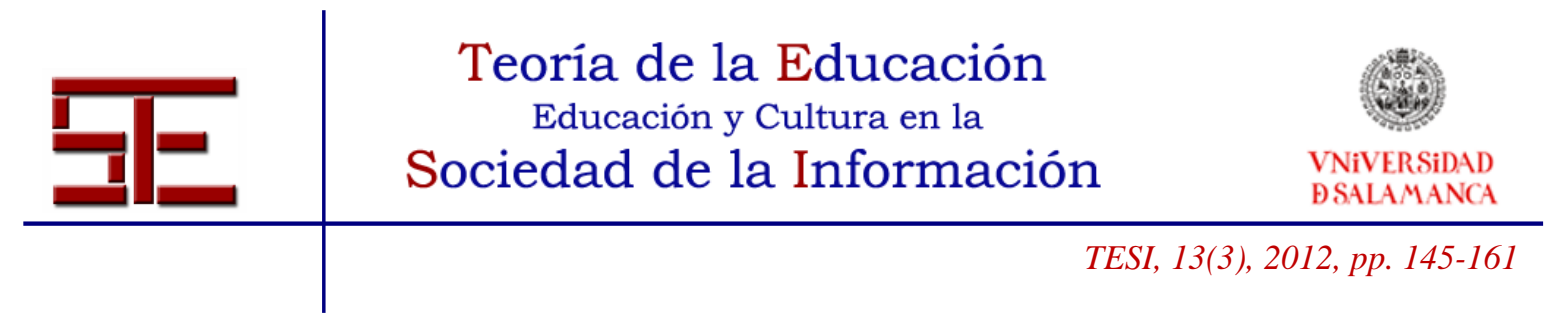

Trujillo, J. (2007, 15 abril). Robótica pedagógica vs Robótica Educativa, mensaje compartido en: http://nticsaeiuaemex.blogspot.com/2007/04/robtica-pedaggica-vsrobtica-educativa.html

Vitko, A., Jurišica, L., Babinec, A., Duchoň, F., \& Kl'účik, M. (2010). Some didactic aspects of teaching robotics. 1-st Slovak-Austrian International Conference on Robotics in Education.

Williams, D., Ma, Y., Prejean, L., Ford, M. J., \& Lai, G. (2008). Acquisition of Physics Content Knowledge and Scientific Inquiry Skills in a Robotics Summer Camp. Journal of Research on Technology in Education, 201-216.

Xuemei, L. Jin-hu, L. \& Lixing, D. (2009). Constructing Innovative Laboratory through Intelligent Learning Objects. En 2009 International Conference on Industrial Mechatronics and Automation. Guangzhou.

Para citar el presente artículo puede utilizar la siguiente referencia:

Ortiz Meza, J. G., Ríos Ramírez, A. y Bustos Gardea, R. A. (2012). Laboratorio móvil tecno educativo: cursos de robótica de bajo costo para la alfabetización científica y tecnológica. Revista Teoría de la Educación: Educación y Cultura en la Sociedad de la Información. 13(3), 145-161 [Fecha de consulta: dd/mm/aaaa].

http://campus.usal.es/ revistas_trabajo/index.php/revistatesi/article/view/9135/9368

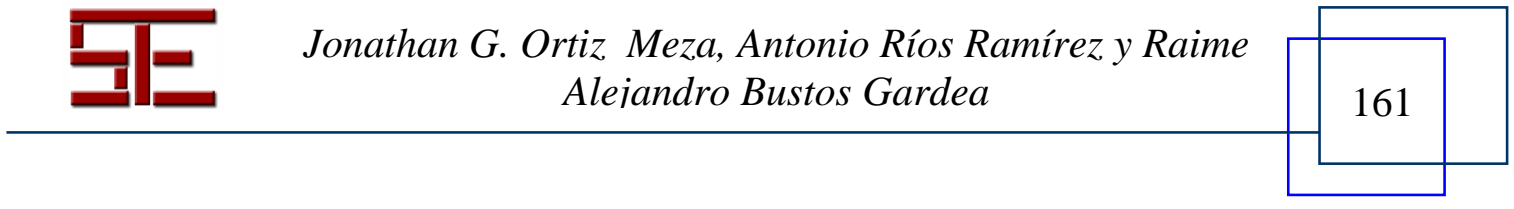

